\title{
SU(2) Gluon Propagator on a Coarse Anisotropic Lattice
}

\author{
Ying Chen ${ }^{2 *}$ Bing $\mathrm{He}^{2}$, He $\mathrm{Lin}^{2}$, Ji-min $\mathrm{Wu}^{1,2}$ \\ ${ }^{1}$ CCAST (World Laboratory), P.O.Box 8730, Beijing 100080, P.R.China \\ ${ }^{2}$ Institute of High Energy Physics, Academia Sinica, P.O.Box 918(4), Beijing 100039, P.R.China ${ }^{\dagger}$
}

October 25, 2018

\begin{abstract}
We calculated the $\mathrm{SU}(2)$ gluon propagator in Landau gauge on an anisotropic coarse lattice with the improved action. The standard and the improved scheme are used to fix the gauge in this work. Even on the coarse lattice the lattice gluon propagator can be well described by a function of the continuous momentum. The effect of the improved gauge fixing scheme is found not to be apparent. Based on the Marenzoni's model, the mass scale and the anomalous dimension are extracted and can be reasonably extrapolated to the continuum limit with the values $\alpha \sim 0.3$ and $M \sim 600 \mathrm{MeV}$. We also extract the physical anisotropy $\xi$ from the gluon propagator due to the explicit $\xi$ dependence of the gluon propagator.
\end{abstract}

PACS: 11.15.Ha, 12.38.Gc

Key words: gluon propagator, anisotropic lattice, improved action

${ }^{*}$ Email address: cheny@hptc5.ihep.ac.cn

${ }^{\dagger}$ permanent address 


\section{Introduction}

The gluon propagator is well defined in the pure non-Abelian gauge theory and is thought of one of the most basic quantities of QCD. Due to the asymptotic freedom, the gluon propagator at small distances (or large momentum) can be described sufficiently by the perturbation theory in which the gluon is expected to propagate as a massless particle. However, any asymptotic gluon states and isolated quarks are absent, and this motivated the confinement mechanism that gluons and quarks are only confined inside hadrons. This physical structure may imply that the gluon propagator is quite different from those of stable particles. To understand the structure of the gluon propagator, especially its infrared behavior, a nonperturbative study of the gluon propagator is required.

Lattice simulation provides a powerful method to study the nonperturbative properties of QCD. The first lattice simulations of the gluon propagator were carried out by Mandula and Ogilvie [1] and by Gupta et al [2] in the late 1980s, in whose studies the gluon propagator in Landau gauge was interpreted in terms of a massive particle propagator. The simulations of Marenzoni et al [3] gave the result that in terms of an anomalous dimension the propagator fell off quite differently from that in perturbation theory. The most recent simulation carried out on a very large lattice by Leinweber et al gave the implication that the gluon propagator should be interpreted in terms of neither a massless particle nor a free massive one [4]. However, even though there has been substantial progress in the lattice simulation of gluon propagator in the last decade, a definitive picture of the gluon propagator has not been obtained yet. For reference see the overview of Mandula [5].

The development of the improved actions is an important progress in lattice QCD in last decade. If the lattice action is properly improved, the costs of the Monte Carlo simulation will be greatly reduced and the numerical study can be performed on much coarser lattice system. Ma [6] simulated the SU(3) gluon propagator in Landau gauge on a coarse lattice using tadpole improved Symanzik's action with substantially reduced lattice artifact. In his work the propagator was fitted using Marenzoni's model and gave the result that the mass scale turns out good scaling behavior and the anomalous dimension can be well extrapolated to the continuum limit $a \rightarrow 0$.

Since the anisotropic lattice has a lot of advantages in the Monte carlo simulation of 
lattice QCD on coarse lattice, it is meaningful to study the behavior of gluon propagator on anisotropic lattice and the effect of this temporal-spatial asymmetric lattice on the gluon propagator because the lattice definition of gluon propagtor is explicitly dependent on the anisotropy ratio $\xi$. Another goal of this work is to explore the efficiency of the improved guage fixing scheme introduced by Bonnet et al [7]. This work is organized as follows. In section II we describe the lattice formulations used in this work. Section III is devoted to the simulation details where we give the mass scales and the anomalous dimensions extracted from the lattice propagator as well as their lattice spacing dependence. In Section IV we discuss the effects of the asymmetric lattice on the gloun propagator and extract the anisotropy ratio for various lattice spacings. Section $\mathrm{V}$ is the summary.

\section{Lattice Formulations}

We use the naive steepest descent method to fix the Landau guage [8] by maximising the usual Landau gauge fixing functional. There are many choices of the definition of the gauge fixing functional. The frequently used version (called standard one in this work) is

$$
\mathcal{F}_{1}^{G}[\{U\}]=\sum_{x, \mu} \frac{1}{2} \operatorname{Tr}\left\{U_{\mu}^{G}(x)+U_{\mu}^{G}(x)^{\dagger}\right\}
$$

where

$$
U_{\mu}^{G}(x) \equiv G(x) U_{\mu}(x) G(x+\hat{\mu})^{\dagger}
$$

and

$$
G(x)=\exp \left\{-i \sum_{a} \omega^{a}(x) T^{a}\right\}
$$

Taking the extremum condition

$$
\frac{\delta \mathcal{F}_{1}^{G}}{\delta \omega^{a}(x)} \propto \sum_{\mu}\left[U_{\mu}^{G}(x-\hat{\mu})-U_{\mu}^{G}-\left(U_{\mu}^{G}(x-\hat{\mu})-U_{\mu}^{G}\right)^{\dagger}\right] \equiv \Delta_{1}(x)=0
$$

and expanding the gauge links, $U_{\mu}(x)=\mathcal{P} \exp \left\{i a g \int_{0}^{1} d t A_{\mu}(x+\hat{\mu} a t)\right\}$, in the small lattice spacing, one obtains the Landau gauge condition on the lattice as

$$
\sum_{\mu} \partial_{\mu} A_{\mu}(x)=\sum_{\mu}\left\{-\frac{a^{2}}{12} \partial_{\mu}^{3} A_{\mu}(x)+O\left(a^{4}\right)\right\} .
$$


The lattice artifact to the gauge condition is of order $O\left(a^{2}\right)$. An alternative gauge fixing functional is introduced by Bonnet et al [7] as follows,

$$
\mathcal{F}_{2}^{G}[\{U\}]=\sum_{x, \mu} \frac{1}{2} \operatorname{Tr}\left\{U_{\mu}^{G}(x) U_{\mu}^{G}(x+\hat{\mu})+\text { h.c. }\right\} .
$$

Similarly the equation

$$
\begin{aligned}
\frac{\delta \mathcal{F}_{2}^{G}}{\delta \omega^{a}(x)} & \propto \sum_{\mu} \operatorname{Tr}\left[U_{\mu}^{G}(x-2 \hat{\mu}) U_{\mu}^{G}(x-\hat{\mu})-U_{\mu}^{G}(x) U_{\mu}^{G}(x+\hat{\mu})-\text { h.c. }\right] \\
& \equiv \Delta_{2}=0
\end{aligned}
$$

gives another realization of lattice Landau gauge,

$$
\sum_{\mu} \partial_{\mu} A_{\mu}(x)=\sum_{\mu}\left\{-\frac{a^{2}}{3} \partial_{\mu}^{3} A_{\mu}(x)+O\left(a^{4}\right)\right\} .
$$

The $O\left(a^{2}\right)$ artifact can be removed by combining the two types of the gauge fixing functional to an improved one [7], $\mathcal{F}_{I m p}^{G} \equiv \frac{4}{3} \mathcal{F}_{1}^{G}-\frac{1}{12 u_{\mu}} \mathcal{F}_{2}^{G}$, where $u_{\mu}$ refers to the tadpole improved parameter of the gauge links in $\mu$ direction. The improved functional gives the following new function

$$
\Delta_{\text {Imp }}(x)=\frac{4}{3} \Delta_{1}(x)-\frac{1}{3 u_{\mu}} \Delta_{2}(x) .
$$

The steepest descents approach is realized by defining the local gauge transformation as

$$
\begin{aligned}
G_{i}(x) & =\exp \left\{\frac{\alpha}{2} \Delta_{i}(x)\right\} \\
& \approx 1+\frac{\alpha}{2} \Delta_{i}(x),
\end{aligned}
$$

where $\alpha$ is a tuneable step-size prameter. The lattice Landau gauge is fixed by decreasing the quantity,

$$
\theta_{i}=\frac{1}{2 V} \sum_{x} \operatorname{Tr}\left\{\Delta_{i}(x) \Delta_{i}(x)^{\dagger}\right\}
$$

to a sufficiently small number. Here the suffix $i$ stands for 1, 2 and Imp.

In this work, the simulations are performed on an $8^{3} \times 24$ anisotropic lattice by using tadpole-improved Symanzik's action [9,10. The lattice spacing in the temporal direction is smaller than that in the spatial direction with the bare anisotropy ratio $\xi_{0}$. The action we use is given as

$$
\begin{aligned}
S_{1} & =\beta \sum_{x, s>s^{\prime}}\left[\frac{5}{3} \frac{P_{s s^{\prime}}}{\xi_{0} u_{s}^{4}}-\frac{1}{12} \frac{R_{s s^{\prime}}}{\xi_{0} u_{s}^{6}}-\frac{1}{12} \frac{R_{s^{\prime} s}}{\xi_{0} u_{s}^{6}}\right] \\
& +\beta \sum_{x, s}\left[\frac{4}{3} \frac{P_{s t} \xi_{0}}{u_{s}^{2} u_{t}^{2}}-\frac{1}{12} \frac{R_{s t} \xi_{0}}{u_{s}^{4} u_{t}^{2}}\right]
\end{aligned}
$$


where $\beta=\frac{4}{g^{2}}, g$ is the gauge coupling. $P_{\mu \nu}$ is the plaquette operator and $R_{\mu \nu} 2 \times 1$ rectangular operator, $u_{s}$ and $u_{t}$ are tadpole improvement parameters. $u_{s}$ is defined by $u_{s}=<(1 / 2) \operatorname{Tr} W_{s p}>^{1 / 4}$, while $u_{t}$ is set to be 1 because of $u_{t}=1-O\left(\xi_{0}^{-2}\right)\left(\xi_{0}\right.$ is much bigger than one).

The continuum gluon propagator $D_{\mu \nu}^{a b}$ in Landau gauge is characterized by a single function $D\left(q^{2}\right)$ as

$$
D_{\mu \nu}^{a b}(q)=\delta_{a b}\left(\delta_{\mu \nu}-\frac{q_{\mu} q_{\nu}}{q^{2}}\right) D\left(q^{2}\right)
$$

where $a$ and $b$ are the color index. The structure of $D\left(q^{2}\right)$ is of physical interest. In the large momentum range $D\left(q^{2}\right)$ takes its perturbative form and is proportional to $1 / q^{2}$, while its infrared behavior may be more complicated and should be reconciled with the confinement. The lattice simulation of the gluon propagator is to determine its structure nonperturbatively. The lattice version of $D\left(q^{2}\right)$ is denoted as $D_{L}(q)$, which can be constructed in terms of the Fourier transfromed vector potential $A_{\mu}(q)$. The vector potential of $\mathrm{SU}(2)$ gauge field is defined from the gauge links

$$
\begin{aligned}
A_{\mu}\left(x+a_{\mu} \hat{\mu} / 2\right) & =\frac{1}{2 i g a_{\mu}}\left(U_{\mu}(x)-U_{\mu}^{\dagger}(x)-\frac{1}{2} \operatorname{Tr}\left(U_{\mu}(x)-U_{\mu}^{\dagger}(x)\right)\right) \\
& \equiv \frac{1}{a_{\mu}} \hat{A}_{\mu}\left(x+a_{\mu} \hat{\mu} / 2\right) .
\end{aligned}
$$

where $a_{\mu}$ is the lattice spacing in $\mu$ direction, and its Fourier transformation is defined as

$$
A_{\mu}(q)=\left(\prod_{\mu} a_{\mu}\right) \sum_{x} e^{-i q \cdot\left(x+a_{\mu} \hat{\mu} / 2\right)} A_{\mu}\left(x+a_{\mu} \hat{\mu} / 2\right) \equiv \frac{1}{a_{\mu}} \hat{A}_{\mu}(q),
$$

where $q$ is the momentum

$$
q_{\mu}=\frac{2 \pi}{L_{\mu} a_{\mu}} n_{\mu}, \quad n_{\mu}=0,1, \ldots, L_{\mu}-1 .
$$

Here the notation $\hat{A}$ is adopted for the convenience of the future discussion. The function $D_{L}(q)$ is defiend as

$$
D_{L}(q)=\frac{2}{9 \prod_{\mu}\left(L_{\mu} a_{\mu}\right)} \sum_{\mu}\langle 0| \operatorname{Tr}\left(A_{\mu}(q) A_{\mu}^{\dagger}(q)|0\rangle,\right.
$$

which approaches to the function $D\left(q^{2}\right)$ in the limit of $a_{\mu} \rightarrow 0$.

\section{Lattice Simulation}

We used the action defined in Eqn.(12) to generate gluonic configurations at $\beta=1.0,1.1,1.2$ on an $8^{3} \times 24$ anisotropic lattice. The samples of configurations and the values of $u_{s}$ and 
$a_{s}$ [11] for various $\beta$ are listed in Table 1. The configurations are separated by 50 Monte carlo sweeps, each of which is composed of 4 heatbath and a microcanonical iterations. We performed the standard gauge fixing and the improved gauge fixing for each configuration respectively and stopped the gauge transfromation at $\theta_{i}<10^{-8}$. The Landau gauge is surely reached for each configuration, for it was observed that for any $q=(\mathbf{q}, 0), \operatorname{tr} A_{4}(q) A_{4}^{\dagger}(q)$ are of order $10^{-6}$ and are about five order smaller than other components.

It is expected that the function $D_{L}(q)$ measured on the lattice approximately depend only on $q^{2}=\sum_{\mu} q_{\mu}^{2}$ for the small enough $a_{\mu} q_{\mu}$. Previous simulations, which were performed on large lattice with the Wilson action, gave the results that this is only true for a small range $a^{2} q^{2}<1$. By applying the improved action we find that the function $D_{L}(q)$ from our simulation behaves well as a function of $q^{2}$ in a much larger momentum range $0<a^{2} q^{2}<9$ which corresponds to almost the full first Brillouin zone (see Figure 1a, 1b, 1c, the data points are the averages of $D_{L}(q)$ with the same $q^{2}$ ). This range corresponds to almost the full first Brillouin zone. From now on we denote $D_{L}(q)$ as $D_{L}\left(q^{2}\right)$.

We use the model [3] to fit the data points of $D_{L}\left(q^{2}\right)$,

$$
D_{L}\left(q^{2}\right)=\frac{Z}{\left(M^{2}\right)^{1+\alpha}+\left(q^{2}\right)^{1+\alpha}},
$$

where $M$ is a dimensional parameter, $\alpha$ the anomalous dimension and $Z$ a parameter related to $g$ and the renormalization constant of wave-function. We perform the fitting in two ways which use the data sets including (fit 1) and excluding (fit 2) the zero momentum point respectively, and the results are listed in Table 2 and Table 3. The quality of both fits are acceptable due to the very small $\chi^{2} /$ d.o.f. It is found that the effect of the improvement of gauge fixing is not apparent, for the fitting results of $D_{L}\left(q^{2}\right)$ through the standard and the improved gauge fixing procedures are almost the same. The mass scale $M$ in physical unit is listed in Table 4. In fit 2, the mass scale is approximately $600 \mathrm{MeV}$ within the errors and insensitive to the lattice spacing, while in fit 1 the differences of $M$ for various lattice spacing are significant.

The anomalous dimension $\alpha$ is extracted from both the fits. In fit 1 the value of $\alpha$ varies with $\beta$ and the $a_{s}$ dependence of $\alpha$ can be well described as

$$
\alpha\left(a_{s}\right)=\alpha_{0}+\alpha_{1} a_{s}^{4},
$$


with

$$
\alpha_{0}=0.309(10) \quad \alpha_{1}=-19.9(1.2) \quad \chi^{2}=0.097 .
$$

In fit 2 , the values of $\alpha$ are averaged to be $\bar{\alpha}=0.282(15)$ and less related to the lattice spacing.

Even though the small discrepancy, both of the fits give the non-zero anomalous dimension $\alpha \sim 0.3$. This value is compatible with the result of Ma's simulation of $\mathrm{SU}(3)$ gluon propagator [6]' $(\alpha=0.285(20))$ but smaller than the results of Marenzoni [3] $(\alpha \sim 0.4-0.6)$.

\section{The Renormalized Anisotropy Ratio}

Since the MC simulation is performed on an anisotropic lattice, there naturally arises a quesiton: what is the effect of this asymmetry of the temporal and the spatial direction on the physical observables and how differently the physical obsevables are affected the renormalized anisotropy ratio from the bare parameter $\xi_{0}$ ( here the bare parameter refers to the parameter in the action which justifies that the lattcie action would take the same form as that of the continuum action at the classical level when the continuum limit $a \rightarrow 0$ is approached, while the renormalized one, $\xi=\xi\left(\xi_{0}, \beta\right)$ is defined by the ratio $a_{s} / a_{t}$ and is a funciton of the bare one and the coupling constant owing to the renormalization effects.) This question has been explored by Klassen et al. who extracted $\xi$ from the quark static potential [12]. We propose here a new scheme to extract the renormalized anisotropy ratio from the gluon propagator measured on the anisotropic lattice.

The lattice version of the funciton $D_{L}\left(q^{2}\right)$ and the continuous momentum are explicitly related to $\xi$ as follows

$$
\begin{aligned}
D_{L}(q) & =\frac{2}{9 \prod_{\mu}\left(L_{\mu} a_{\mu}\right.} \sum_{\mu}\langle 0| \operatorname{Tr}\left(A_{\mu}(q) A_{\mu}^{\dagger}(q)|0\rangle\right. \\
& \propto\left\langle 0\left|\operatorname{Tr}\left(\sum_{i=1}^{3} \hat{A}_{i}(q) \hat{A}_{i}^{\dagger}+\xi^{2} \hat{A}_{4}(q) \hat{A}_{4}^{\dagger}\right)\right| 0\right\rangle \\
& \equiv \hat{D}_{L}(q, \xi) .
\end{aligned}
$$

and

$$
q^{2}=\left(\frac{2 \pi}{L_{s} a_{s}}\right)^{2}\left(\sum_{i} n_{i}^{2}+\frac{\xi^{2}}{9} n_{4}^{2}\right)
$$


In practice, the renormalized anisotropy $\xi$ is unknown at the beginning of the calculation and the measured quantity is $D_{L}\left(q, \xi_{0}=3\right)$. For convenience we introduce a notation $\hat{q}^{2}=\sum_{\mu} n_{\mu}^{2}$ where $n_{\mu}$ is defined in the Eqn.(16). If the function $D_{L}(q)$ is a function of $q^{2}$ and $\xi / \xi_{0}=1$, the rotational invariance requires that $D_{L}(q)$ take the same value at all the momentum with the same $\hat{q}^{2}$. From the analysis of our results we found that this is only correct for the momenuta with the same $\hat{q}^{2}$ and the same $n_{4}$. For those momenta with the same $\hat{q}^{2}$ but different $n_{4}$, the difference of $D_{L}(q)$ is significant. This discrepancy can be attributed to the fact that $\xi / \xi_{0} \neq 1$, as can be found in the following.

With the conjecture that $\epsilon / \xi_{0}$ is small, only the lowest order term of $\epsilon=\xi_{0}-\xi$ is relevant in the discussion, $D_{L}\left(q, \xi_{0}=3\right)$ is related to the expected $D_{L}(q, \xi)$ as follows,

$$
\hat{D}_{L}(q, \xi)=\hat{D}_{L}(q, 3-\epsilon)=\hat{D}_{L}(q, 3)-6 \epsilon\left\langle 0\left|\operatorname{Tr} \hat{A}_{4}(q) \hat{A}_{4}^{\dagger}\right| 0\right\rangle+O\left(\epsilon^{2}\right)
$$

For simplicity we define

$$
\alpha(q)=\left\langle 0\left|\operatorname{Tr} A_{4}(q) A_{4}(q)^{\dagger}\right| 0\right\rangle
$$

Another reason for the discrepancy is the $\xi$ dependence of the continuous momentum also introduces a small shift $\Delta q^{2}$ of momentum square between two momenta $q$ and $q^{\prime}$ with the same $\hat{q}^{2}$. With both the two factors in consideration and accepting the fact that $D_{L}(q, \xi)$ is well described by a function of $q^{2}$, this discrepancy, described by $\eta=\frac{\hat{D}_{L}\left(q^{\prime}, 3\right)}{\hat{D}_{L}(q, 3)}$, can be re-expressed in the lowest order of $\epsilon=\xi$,

$$
\begin{aligned}
\eta & =\frac{\hat{D}_{L}\left(q^{\prime}, 3\right)}{\hat{D}_{L}(q, 3)}=\frac{\hat{D}_{L}\left(q^{\prime}, \xi\right)+6 \epsilon \alpha\left(q^{\prime}\right)}{\hat{D}_{L}(q, 3)+6 \epsilon \alpha(q)} \\
& =\frac{\hat{D}_{L}\left(q^{\prime}, \xi\right)}{\hat{D}_{L}(q, \xi)}\left[1+6 \epsilon\left(\frac{\alpha\left(q^{\prime}\right)}{\hat{D}_{L}\left(q^{\prime}, \xi\right)}-\frac{\alpha(q)}{\hat{D}_{L}(q, \xi)}\right)\right] \\
& =\left(1-\left.\Delta q^{2} \frac{d}{d q^{2}} \ln \hat{D}_{L}\left(q^{2}, \xi\right)\right|_{q^{2}=q^{\prime 2}}\right)\left[1+\frac{6 \epsilon}{\hat{D}_{L}(q, \xi)}\left(\frac{\hat{D}_{L}(q, \xi)}{\hat{D}_{L}\left(q^{\prime}, \xi\right)} \alpha\left(q^{\prime}\right)-\alpha(q)\right)\right] .
\end{aligned}
$$

Since only the lowest order of $\epsilon$ is considered, after replacing approximately the ratio $\frac{\hat{D}_{L}\left(q^{\prime}, \xi\right)}{\hat{D}_{L}(q, \xi)}$ with $1 / \eta$ and rearranging the Eqn.(24), the quantity $\epsilon$ can be expressed by the quantities which can be measured directly from the lattice simulation (see the following equation),

$$
\epsilon\left(q^{2}\right)=\frac{1-\eta}{\frac{2}{3} \delta\left(q^{2}\right) \frac{d}{d q^{2}} \ln \hat{D}_{L}(q, \xi)+\frac{6}{\hat{D}_{L}(q, \xi)}\left(\alpha(q)-\frac{1}{\eta} \alpha\left(q^{\prime}\right)\right)} .
$$

Here the momenta $q$ and $q^{\prime}$ satisfy $\hat{q}^{2}=\left({\hat{q^{\prime}}}^{2}\right.$ with $\delta\left(q^{2}\right)=\frac{2}{3} \epsilon\left(\left(q^{\prime}\right)_{4}^{2}-q_{4}^{2}\right)$. The results of $\epsilon\left(q^{2}\right)$ for $\beta=1.0,1.1,1.2$ are listed in Table . Theoretically, $\xi / \xi_{0}$ should be independent of $q^{2}$. 
From the Table it is found that the measured $\xi / \xi_{0}$ are insensitive to $q^{2}$ for most momenta. However, a few irregular values such as $q^{2}\left(\delta\left(q^{2}\right)=6(1)\right.$ for $\beta=1.0, q^{2}\left(\delta\left(q^{2}\right)=4(1)\right.$ for $\beta=1.1$ and $q^{2}\left(\delta\left(q^{2}\right)=4(1)\right.$ for $\beta=1.2$, are a bit larger than the others. We attribute it to the reason that the rotational invariance is violated to some extent so that the value of $D_{L}(q)$ with momenta directed along one of the four axes is different from those with momenta directed off-axis. Averaging the regular values we obtain

$$
\begin{aligned}
& \xi / \xi_{0}=1.23 \quad \text { for } \quad \beta=1.0 \\
& \xi / \xi_{0}=1.15 \quad \text { for } \quad \beta=1.1 \\
& \xi / \xi_{0}=1.16 \quad \text { for } \quad \beta=1.2 \text {. }
\end{aligned}
$$

These are raw results at the lowest order of $\epsilon$ and we do not plan to extract the $\beta$ dependence of $\xi / \xi_{0}$ from only three data points. What we can conclude at present is that the renormalization effects for the anisotropy ratio is significant and our results is consistent with those from the study of static quark potential by Klassen et al [12]. Morningstar et al [13] have simulated the $\mathrm{SU}(3)$ glueball mass on the anisotropic lattice with the same action as that we use and reported that $\xi / \xi_{0}$ extracted from the static quark potential is apporximately one within few percents discrepancy. Work is underway to explain the reason why the anisotropy ratios extraceted from different quantities do not comply with each other.

\section{Summary}

We studied the $\mathrm{SU}(2)$ gluon propagator in Landau gauge on an $8^{3} \times 24$ anisotropic lattice by using the tadpole improved Symanzik's action. Although the improvement scheme is also used to fix the gauge, we found that its effect is not significant, while the lattice artifact is significantly suppressed by using the improved action. The function $D_{L}(q)$ in the propagator can be well described as a function of continuous momentum square $q^{2}$ even in the full first Brillouin zone. We used the model suggested by Marenzoni et al to fit the function $D_{L}(q)$ and extracted the mass scale and the anomalous dimension therein. It is found that the value of the mass scale is approximately independent of the lattice spacing and the anomalous dimension can be well extrapolated to the value about 0.3 in the continuum limit.

The effects of the asymmetric lattice on the gluon propagator are also explored in this

work. Based on the fact that the lattice definition of the gluon propagator is related to the 
renormalized anisotropy ratio $\xi=a_{s} / a_{t}$, we deduced a formula to measure the $\xi$ nonperturbatively at various momentum. Even in the lowest order of $\epsilon=\xi_{0}-\xi$, the measured values of $\xi$ are insensitive to the momentum and are consistent with the results extracted from the static quark potential.

\section{Acknowledgement}

This work is supported by the Natural Science Foundation of China under the Grant No. 19677205 and No. 19991487, and by the National Research Center for Intelligent Computing System under the contract No. 99128.

\section{References}

[1] J. E. Mandula and M. Ogilvie, Phys. Lett. B185(1987)127.

[2] R. Gupta, G. Guralnik, G. Kilcup, A. Patel, S. Sharpe, T. Warnock, Phys. Rev. D36(1987)2813.

[3] P. Marenzoni et al., Phys. Lett. B318 (1993)511; P. Marenzoni, G. Martinelli, and N. Stella, Nucl. Phys. B455(1995) 339.

[4] Leinweber, Skullerud, Williams, and Parrinello, Phys. Rev. D (Rapid Commun.)58 (1998) 031501-1, hep-lat/9811027.

[5] J. E. Mandula, Phys. Rep. 315 (1999) 273.

[6] J. P. Ma, Mod. Phys. Lett. A15 (2000)229, hep-lat/9903009.

[7] F. D. R. Bonnet, P. U. Bowman et al, Austral. J. Phys. 52 (1999)939, hep-lat9905006.

[8] C. T. H. Davies et al, Phys. Rev. D37(1988)1581.

[9] C. J. Morningstar and M. Peardon, Nucl. Phys. B(Proc. Suppl.)47(1996)258.

[10] G. P. Lepage, P. B. Mackenzie, Phys. Rev. D48(1993)2250.

[11] J. B. Zhang, M. Jin and D. R. Ji, Chin. Phys. Lett. 15(1998)865; Y. Chen, B. He, H. Lin and J. Wu, Commun. Theor. Phys.(in press). 
[12] T. R. Klassen, Nucl. Phys. B533(1998) 557.

[13] C. Morningstar, M. Peardon, Phys. Rev. D60 (1999) 034509, hep-lat9901004.

\section{Tables and Figures}

\begin{tabular}{|c|c|c|c|}
\hline$\beta$ & $u_{s}$ & $a_{s}(\mathrm{fm})$ & No. of Configs. \\
\hline 1.0 & 0.8215 & 0.277 & 200 \\
\hline 1.1 & 0.8365 & 0.233 & 200 \\
\hline 1.2 & 0.8593 & 0.183 & 200 \\
\hline
\end{tabular}

Table 1: The parameters used in the simulations of this work.

\begin{tabular}{|c|c|c|c|c|c|c|}
\hline & \multicolumn{3}{|c|}{ Standard fixing } & \multicolumn{3}{c|}{ Improved fixing } \\
\hline$\beta$ & $a_{s}^{2} M^{2}$ & $\alpha$ & $\chi^{2} / d o f$ & $a_{s}^{2} M^{2}$ & $\alpha$ & $\chi^{2} /$ dof \\
\hline 1.0 & $0.552(24)$ & $0.191(15)$ & 0.17 & $0.547(24)$ & $0.203(15)$ & 0.17 \\
\hline 1.1 & $0.357(14)$ & $0.254(13)$ & 0.17 & $0.360(14)$ & $0.268(13)$ & 0.15 \\
\hline 1.2 & $0.311(12)$ & $0.285(13)$ & 0.13 & $0.313(11)$ & $0.298(13)$ & 0.12 \\
\hline
\end{tabular}

Table 2: The results of the fit 1 which includes the zero-momentum data point. $\alpha$ and $a_{s}^{2} M^{2}$ in the propagator model are extracted at various value of $\beta$. The data illustrate that the effect of the improved gauge fixing scheme is not apparent.

\begin{tabular}{|c|c|c|c|c|c|c|}
\hline & \multicolumn{3}{|c|}{ Standard fixing } & \multicolumn{3}{c|}{ Improved fixing } \\
\hline$\beta$ & $a_{s}^{2} M^{2}$ & $\alpha$ & $\chi^{2} / d o f$ & $a_{s}^{2} M^{2}$ & $\alpha$ & $\chi^{2} / d o f$ \\
\hline 1.0 & $0.808(95)$ & $0.273(33)$ & 0.11 & $0.807(88)$ & $0.288(32)$ & 0.11 \\
\hline 1.1 & $0.454(70)$ & $0.289(28)$ & 0.18 & $0.439(69)$ & $0.297(28)$ & 0.17 \\
\hline 1.2 & $0.309(66)$ & $0.284(27)$ & 0.15 & $0.317(66)$ & $0.299(27)$ & 0.13 \\
\hline
\end{tabular}

Table 3: The results of the fit 1 which excludes the zero-momentum data point. $\alpha$ and $a_{s}^{2} M^{2}$ in the propagator model are extracted at various value of $\beta$. The data illustrate that the effect of the improved gauge fixing scheme is not apparent. 


\begin{tabular}{|c|c|c|c|}
\hline$\beta$ & 1.0 & 1.1 & 1.2 \\
\hline $\mathrm{M}(\mathrm{MeV})($ Fit 1) & $529(12)$ & $506(10)$ & $601(12)$ \\
\hline $\mathrm{M}(\mathrm{MeV})($ Fit 2) & $640(37)$ & $571(44)$ & $599(64)$ \\
\hline
\end{tabular}

Table 4: Tha mass scales in physical unit. In fit 2, within the range of errors, the mass scale takes approximately the same value and is independent of the lattice spacing.

\begin{tabular}{|c|c|c|c|c|c|c|}
\hline & \multicolumn{2}{|c|}{$\beta=1.0$} & \multicolumn{2}{c|}{$\beta=1.1$} & \multicolumn{2}{c|}{$\beta=1.2$} \\
\hline$q^{2}\left(\delta\left(q_{4}^{2}\right)\right)$ & $\epsilon$ & $\xi / \xi_{0}$ & $\epsilon$ & $\xi / \xi_{0}$ & $\epsilon$ & $\xi / \xi_{0}$ \\
\hline $1(1)$ & -1.04 & 1.35 & -0.31 & 1.10 & -0.40 & 1.13 \\
\hline $2(1)$ & -0.69 & 1.23 & -0.52 & 1.17 & -0.50 & 1.17 \\
\hline $3(1)$ & -0.79 & 1.26 & -0.32 & 1.11 & -0.27 & 1.09 \\
\hline $4(1)$ & -0.66 & 1.22 & -1.14 & 1.38 & -1.09 & 1.36 \\
\hline $4(4)$ & -0.54 & 1.18 & -0.40 & 1.13 & -0.39 & 1.13 \\
\hline $5(1)$ & -0.75 & 1.25 & -0.40 & 1.13 & -0.50 & 1.17 \\
\hline $5(4)$ & -0.63 & 1.21 & -0.41 & 1.14 & -0.43 & 1.14 \\
\hline $6(1)$ & -1.09 & 1.36 & -0.53 & 1.18 & -0.58 & 1.19 \\
\hline $6(4)$ & -0.68 & 1.23 & -0.50 & 1.17 & -0.49 & 1.16 \\
\hline $7(1)$ & -0.85 & 1.28 & -0.44 & 1.15 & -0.62 & 1.21 \\
\hline $8(4)$ & -0.71 & 1.24 & -0.63 & 1.21 & -0.60 & 1.20 \\
\hline
\end{tabular}

Table 5: The difference of the renormalized anisotropy ratio and the bare one $\epsilon=\xi_{0}-\xi$ is measured at various $\hat{q}^{2}$. The ratio $\xi / \xi_{0}$ is insensitive to the momentum except for a few irregular values. These irregular values may be the result of the significant violation of the rotational invariant. 

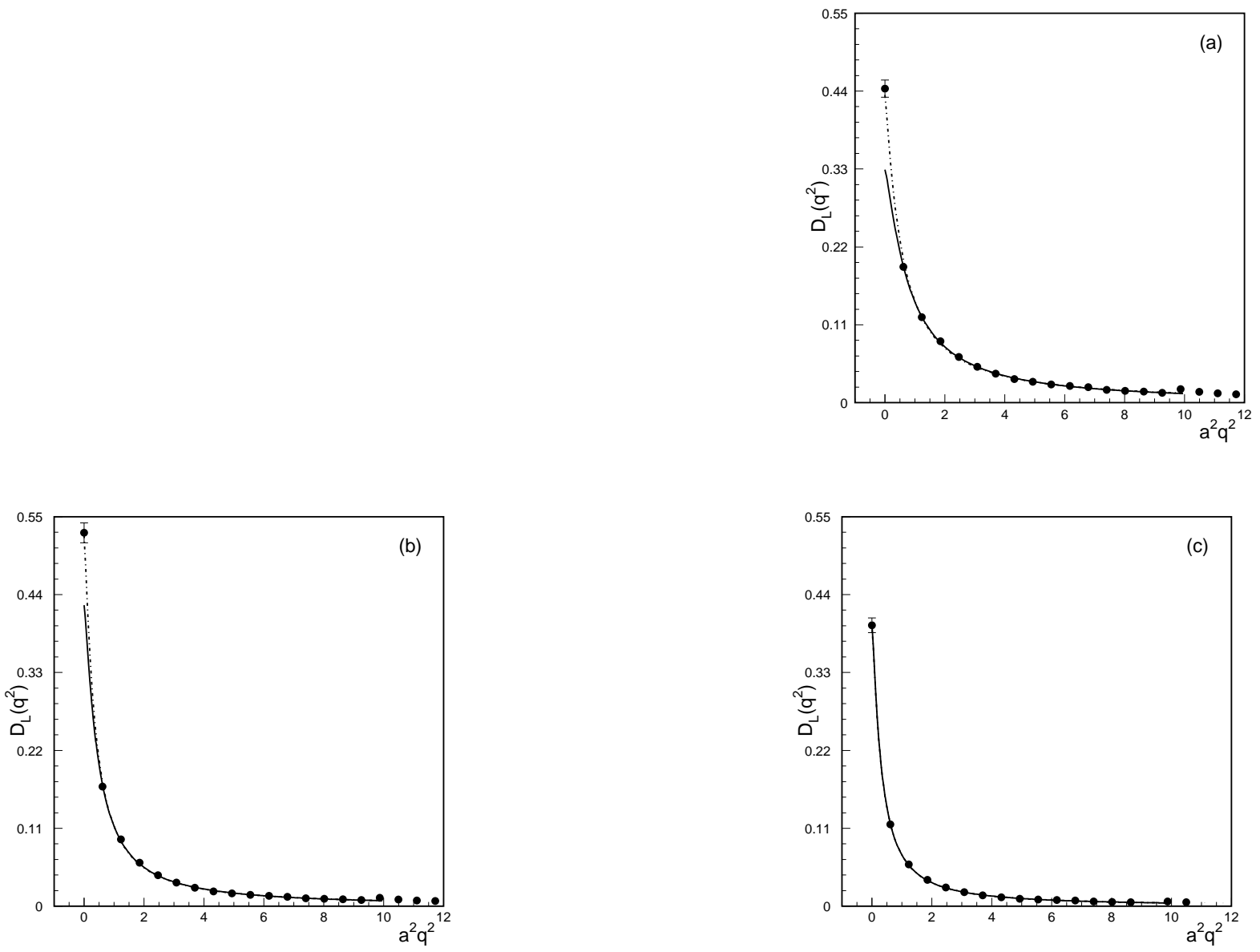

Figure 1: The lattice gluon propagator $D_{L}(q)$ versus the dimensionless continuous momentum $a^{2} q^{2}$ measured at various $\beta$. The filled circles are the data points from the simulation, the dash line shows the result of fit 1 and the full line the results of fit 2 . a) $\beta=1.0$; b) $\beta=1.1$; c) $\beta=1.2$. 


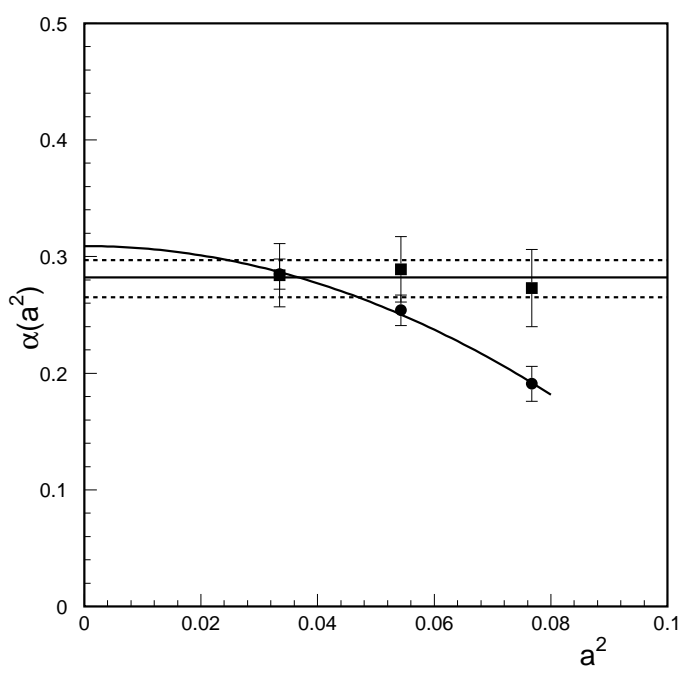

Figure 2: The lattice spacing dependence of the anomalous dimension $\alpha$ is illustrate in the plot. $\alpha$ through fit 1 (filled circles) can be extrapolated to the continuum limit by the model $\alpha(a)=0.309-19.9 a^{4}$ with the $\chi^{2}=0.097$ while $\alpha$ through fit 2 (filled square) is approximately independent of the lattice spacing within the errors. 